Homology, Homotopy and Applications, vol. 7(3), 2005, pp.1-10

\title{
ON REDUCTION MAP FOR ÉTALE $K$-THEORY OF CURVES
}

\author{
G. BANASZAK, W. GAJDA AND P. KRASOŃ \\ (communicated by J.F. Jardine)
}

\begin{abstract}
In this paper we investigate reduction of nontorsion elements in the étale $K$-theory of a curve $X$ over a global field $F$. We show that the reduction map can be well understood in terms of Galois cohomology of $l$-adic representations.
\end{abstract}

Dedicated to Victor Snaith on the occasion of his 60-th birthday.

\section{Introduction}

Assume that $X$ is a smooth, proper and geometrically irreducible curve of genus $g$, defined over a global field $F$. Let $l$ be an odd rational prime different from the characteristic of the field $F$. In the function field case we fix the set of places at infinity. Let $n>0$ and let $S_{l}$ be the set of places of $F$ which consists of places of bad reduction of $X$, places at infinity, and in the number field case, primes lying over $l$. Denote by $\mathcal{X}$ a smooth and proper model of $X$ over the ring of $S_{l}$-integers of $F$. Let $K_{i}^{\text {et }}(Y)$ denote the étale K-theory group of a scheme $Y$ over $S p e c \mathbb{Z}\left[\frac{1}{l}\right]$ cf. [DF]. For places $v \notin S_{l}$, we consider the reduction map:

$$
K_{2 n}^{e t}(\mathcal{X}) \rightarrow K_{2 n}^{e t}\left(\mathcal{X}_{v}\right)
$$

induced on étale $\mathrm{K}$-theory by the injection $\mathcal{X}_{v} \hookrightarrow \mathcal{X}$ of the special fiber at $v$.

Theorem . Let $J$ be the Jacobian variety of $X$ and assume that End $(J)=\mathbb{Z}$. Let $P_{1}, P_{2}, \ldots, P_{r} \in K_{2 n}^{e t}(\mathcal{X})$ be nontorsion elements which are linearly independent over $\mathbb{Z}_{l}$. Fix a partition $\{1,2, \ldots, r\}=I \cup J$ and let $l^{M}$ be a power of $l$. There exist infinitely many primes $v \notin S_{l}$ such that $P_{i}$, for each $i \in I$, reduces in $K_{2 n}^{e t}\left(\mathcal{X}_{v}\right)$ to an element of order at least $l^{M}$ and $P_{j}$, for each $j \in J$, reduces in $K_{2 n}^{e t}\left(\mathcal{X}_{v}\right)$ to zero.

The authors were supported by the KBN grant 1 P03A 027 27. The second author would like to thank Mathematics Department of EPFL in Lausanne for the invitation and hospitality during the visit in summer 2004.

Received October 29, 2004, revised May 19, 2005; published on November 12, 2005.

2000 Mathematics Subject Classification: 11G30, 11S25.

Key words and phrases: Galois cohomology, l-adic representation, étale $K$-theory of a curve. (C) 2005, G. Banaszak, W. Gajda and P. Krasoń. Permission to copy for private use granted. 
The assumption that $\operatorname{End}(J)=\mathbb{Z}$, in the theorem, is implied by the fact that $K_{2 n}^{e t}(\mathcal{X})$ does not support, for $n>0$, any suitable action of $\operatorname{End}(J)$ bigger than $\mathbb{Z}$. In our previous work on the reduction map for abelian varieties [BGK3] we investigated in the present context the maps

$$
J(F) \rightarrow J_{v}\left(k_{v}\right)
$$

for $v \notin S_{l}$ where $J_{v}$ is the Jacobian of $\mathcal{X}_{v}$ and $J(F)$ (resp. $J_{v}\left(k_{v}\right)$ ) is the group of $F$ (resp. $k_{v}$ ) points of $J$ (resp. $J_{v}$ ). This map is an essential part of the map

$$
K_{0}(\mathcal{X}) \rightarrow K_{0}\left(\mathcal{X}_{v}\right)
$$

Note that according to the Beilinson conjectures the Quillen K-group $K_{m}(\mathcal{X})$ is expected to be finitely generated. The Quillen-Lichtenbaum conjecture (cf. [FW] p. $57)$ predicts that the Dwyer-Friedlander map $[\mathrm{DF}]$ :

$$
K_{m}\left(X ; \mathbb{Z} / l^{k}\right) \rightarrow K_{m}^{e t}\left(X ; \mathbb{Z} / l^{k}\right)
$$

is an isomorphism for $m>3$ since the cohomological dimension of $X$ is no bigger than 4 by [Mi], Corrollary 1.4, p 221. Hence by the localization sequences in Ktheory the conjectures imply that for $m>3$ there is an isomorphism:

$$
K_{m}(\mathcal{X}) \otimes_{\mathbb{Z}} \mathbb{Z}_{l} \rightarrow K_{m}^{e t}(\mathcal{X}) .
$$

Provided these conjectures hold true, our Theorem implies the corresponding statement for the even dimensional Quillen K-groups of $\mathcal{X}$. Note that if $X$ is a curve over an algebraically closed field then the Quillen-Lichtenbaum conjecture is supposed to hold for $m \geqslant 2$ since the cohomological dimension of $X$ is no bigger than 2 cf. [Mi] Remark 1.5, p.222.

In section 1 of the paper we introduce intermediate Jacobians and Kummer theory for $l$-adic representations which will be used in section 2 in the proof of the Theorem.

\section{Kummer Theory and intermediate Jacobians}

Let $\bar{F}$ be a fixed separable closure of $F$. For a finite place $v$ in $F$ and a finite extension $L / F$ contained in $\bar{F}$, let $w$ be a finite prime in $L$ over $v$. To indicate that $w$ is not over any place in the set $S_{l}$, we will write $w \notin S_{l}$.

Let $T_{l}$ be a free $\mathbb{Z}_{l}$-module of finite rank $d$. Let $V_{l}=T_{l} \otimes_{\mathbb{Z}_{l}} \mathbb{Q}_{l}$ and $A_{l}=V_{l} / T_{l}$. Consider an $l$-adic representation $\rho_{l}: G_{F} \rightarrow G L\left(T_{l}\right)$ unramified outside $S_{l}$. For $w \notin S_{l}$ we denote by $G_{w}$ the absolute Galois group $G\left(\bar{L}_{w} / L_{w}\right)$ of the completion $L_{w}$ of $L$ at $w$. Let $k_{w}$ be the residue field for the place $w$ and $g_{w}=G\left(\bar{k}_{w} / k_{w}\right)$. The Galois group $g_{w}$ has a topological generator $F r_{w}: k_{w} \rightarrow k_{w}$ defined by the formula $F_{w}(x)=x^{q_{w}}$, where $q_{w}=\left|k_{w}\right|$. The inertia group $I_{w}$ is defined as the kernel of the natural map $G_{w} \rightarrow g_{w}$. We put $H_{f}^{1}\left(G_{w} ; T_{l}\right)=i_{w}^{-1} H_{f}^{1}\left(G_{w} ; V_{l}\right)$, where

$$
i_{w}: H^{1}\left(G_{w} ; T_{l}\right) \rightarrow H^{1}\left(G_{w} ; V_{l}\right)
$$


and the group $H_{f}^{1}\left(G_{w} ; V_{l}\right)$ is defined as follows:

$$
H_{f}^{1}\left(G_{w} ; V_{l}\right)=\operatorname{Ker}\left(H^{1}\left(G_{w} ; V_{l}\right) \stackrel{\operatorname{Res}_{w}}{\longrightarrow} H^{1}\left(I_{w} ; V_{l}\right)\right)
$$

where $I_{w}$ is the inertia subgroup of $G_{w}$. Define

$$
H_{f, S_{l}}^{1}\left(G_{L} ; T_{l}\right)
$$

to be the kernel of the natural map:

$$
H^{1}\left(G_{L} ; T_{l}\right) \rightarrow \prod_{w \notin S_{l}} H^{1}\left(G_{w} ; T_{l}\right) / H_{f}^{1}\left(G_{w} ; T_{l}\right)
$$

Note that as far as $H^{1}$ is concerned, the $l$-adic cohomology is the same as the continuous cohomology. This follows from the simple "lim ${ }^{1}$ argument", since $T_{l} / l^{k} T_{l}$ is finite.

As in [BGK2, Definition 2.3] we define the intermediate Jacobian:

$$
J_{f, S_{l}}\left(T_{l}\right)=\underset{L / F}{\lim _{f, S_{l}}} H_{L}^{1}\left(G_{L} ; T_{l}\right) .
$$

To understand the action of $G_{F}$ on $J_{f, S_{l}}\left(T_{l}\right)$ note that for $L / F$ Galois we have a standard $G_{F}$-action on continuous cochains defining $H^{n}\left(G_{L}, T_{l}\right)$ which induces the action on $H^{n}\left(G_{L}, T_{l}\right)$, in particular on $H^{1}\left(G_{L}, T_{l}\right)$. Similarly, with a little more care, one sees that $G_{F}$ acts on $\prod_{w \notin S_{l}} H^{1}\left(G_{w} ; T_{l}\right) / H_{f}^{1}\left(G_{w} ; T_{l}\right)$. These actions are compatible in the sense that the map defining $H_{f, S_{l}}^{1}\left(G_{L} ; T_{l}\right)$ is $G_{F}$-equivariant. In addition, the corresponding $G_{F}$-actions are compatible with direct limits yielding a $G_{F}$-action on the intermediate Jacobian.

Proposition 1.1. Assume that for any finite extension $L / F$ and any place $w$ of $\mathcal{O}_{L}, w \notin S_{l}$, we have $T_{l}^{F r_{w}}=0$. The following statements hold.

1 The group $J_{f, S_{l}}\left(T_{l}\right)$ is divisible.

2 There is an isomorphism of $G_{F}$-modules $J_{f, S_{l}}\left(T_{l}\right)_{\text {tor }} \cong A_{l}$

$3 H^{1}\left(G_{L} ; T_{l}\right) \cong J\left(T_{l}\right)^{G_{L}}$,

Proof. For the number field case it is [BGK2, Prop. 2.14, Prop. 2.16]. In the function field case the same argument works.

Proposition 1.1 shows that we can do Kummer theory in the $G_{F}$-module $J_{f, S_{l}}\left(T_{l}\right)$. Note that by definition of $A_{l}$ there is a compatible in $k, G_{F}$ - isomorphism $T_{l} / l^{k} T_{l} \cong A_{l}\left[l^{k}\right]$. For any $k>0$ we consider the residual representation

$$
\overline{\rho_{l^{k}}}: G_{F} \rightarrow G L\left(A_{l}\left[l^{k}\right]\right),
$$

induced by $\rho_{l}$. Let $F_{l^{k}}=F\left(A_{l}\left[l^{k}\right]\right)$ denote the global field $\bar{F}^{k e r \overline{\rho_{l^{k}}}}$. For $\hat{P} \in H_{f, S_{l}}^{1}\left(G_{F} ; T_{l}\right)$ we have the Kummer maps [BGK3, (2.3)]:

$$
\phi_{\hat{P}}^{(k)}: G\left(\bar{F} / F_{l^{k}}\right) \longrightarrow A_{l}\left[l^{k}\right]
$$




$$
\phi_{\hat{P}}^{(k)}(\sigma)=\sigma\left(\frac{1}{l^{k}} \hat{P}\right)-\frac{1}{l^{k}} \hat{P},
$$

which are well defined due to Proposition 1.1 (1), (2) and due to the fact that $G\left(\bar{F} / F_{l^{k}}\right)$ acts trivially on $A_{l}\left[l^{k}\right]$ by definition of $F_{l^{k}}$. These maps are compatible with the natural maps $G\left(\bar{F} / F_{l^{k+1}}\right) \rightarrow G\left(\bar{F} / F_{l^{k}}\right)$, therefore they induce a map:

$$
\phi_{\hat{P}}^{(\infty)}: G\left(\bar{F} / F_{l \infty}\right) \longrightarrow T_{l},
$$

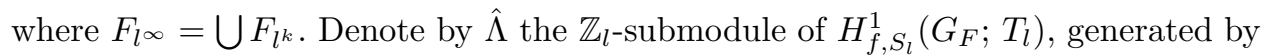
$\hat{P}_{1}, \ldots, \hat{P}_{r}$ and define

$$
\begin{gathered}
\Phi_{\hat{\Lambda}}^{(k)}: G\left(\bar{F} / F_{l^{k}}\right) \longrightarrow \bigoplus_{i=1}^{r} A_{l}\left[l^{k}\right] \\
\Phi_{\hat{\Lambda}}^{(k)}=\left(\phi_{\hat{P}_{1}}^{k}, \ldots, \phi_{\hat{P}_{r}}^{k}\right) .
\end{gathered}
$$

Similarly define

$$
\begin{gathered}
\Phi_{\hat{\Lambda}}^{(\infty)}: G\left(\bar{F} / F_{l}\right) \longrightarrow \bigoplus_{i=1}^{r} T_{l} \\
\Phi_{\hat{\Lambda}}^{(\infty)}=\left(\phi_{\hat{P}_{1}}^{\infty}, \ldots, \phi_{\hat{P}_{r}}^{\infty}\right) .
\end{gathered}
$$

We use the notation $T_{l}^{r}=\bigoplus_{i=1}^{r} T_{l}$. Let $\mathcal{O}$ be a ring with identity which is a finitely generated $\mathbb{Z}$-module. Assume that the ring $\mathcal{O}$ acts on $T_{l}$ and the actions of $\mathcal{O}$ and $G_{F}$ on $T_{l}$ commute. Assume also that $\hat{\Lambda}$ is $\mathcal{O}-$ equivariant and that $\hat{P}_{1}, \ldots, \hat{P}_{r}$ are independent over $\mathcal{O} \otimes_{\mathbb{Z}} \mathbb{Z}_{l}$. In [BGK3, Lemmas 2.12 and 2.13] we proved the following generalization of the theorem of Bertrand [Be, Theorem 2].

Proposition 1.3. Let $G_{l_{\infty}}=G\left(F_{l^{\infty}} / F\right)$ and $V_{l}$ be a semisimple $\mathbb{Q}_{l}\left[G_{l^{\infty}}\right]$-module such that the group $H^{1}\left(G_{l} ; V_{l}\right)$ vanishes. Then the image of $\Phi_{\hat{\Lambda}}^{(\infty)}$ is an open subset of $T_{l}^{r}$ in the l-adic topology.

Observe that by definitions $G_{F}$ acts on $V_{l}$ via its quotient $G_{l \infty}$.

Note that the proofs of Lemmas 2.12 and 2.13 in [BGK3] were done for the number field case, but the function field case can be treated analogously.

\section{Proof of the theorem}

Let $G_{F, S_{l}}$ be the Galois group of the maximal, unramified outside $S_{l}$, extension of $F$. The diagrams (3.1) and (3.2) of [BGK1], for $v \notin S_{l}$ give the following commutative diagram:

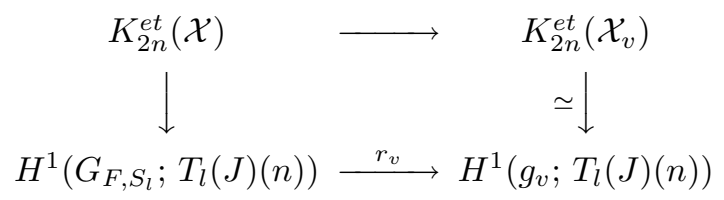


in which the left vertical arrow is surjective with finite kernel and the right vertical arrow is an isomorphism. Let $\hat{P}_{i}$ denote the image of $P_{i}$ via the left vertical arrow of (2.1). In order to prove the Theorem it is enough to show the corresponding statement in Galois cohomology for the reduction map $r_{v}$ of the diagram (2.1). Now we apply the results of section 1 for $T_{l}=T_{l}(J)(n)$ and $\mathcal{O}=\mathbb{Z}$. Observe that all assumptions imposed on $T_{l}$ in section 1 are fulfilled. Namely, by Ogg-Shafarevich criterion cf. [ST], Theorem 1 the set of primes $S_{l}$ contains primes of bad reduction for $J$. Moreover, $T_{l}^{F r_{w}}=0$ for $w \notin S_{l}$ by the Weil conjectures for $J$ proven in [De]. Note that by the Weil conjectures, all eigenvalues of $\operatorname{Fr}_{w}$ acting on $T_{l}(J)$ have absolute values $q_{w}^{\frac{1}{2}}$, and $F r_{w}$ acts on $\mathbb{Z}_{l}(n)$ via $q_{w}^{n}$. Moreover, the $\mathbb{Q}_{l}\left[G_{F}\right]$-module $V_{l}$ is semisimple in the number field case by $[\mathrm{Fa}]$ and in the function field case by [Za1], [Za2]. In addition, in both cases, $H^{1}\left(G_{l^{\infty}} ; T_{l}\right)$ is finite by [Se, Cor. p. 734, and remarque 2, p. 734], for $n=0$ and [J1, p. 338] for $n>0$. Note that for $T_{l}=T_{l}(J)(n)$ we have $H_{f, S_{l}}^{1}\left(G_{L} ; T_{l}\right)=H^{1}\left(G_{L, S_{l}} ; T_{l}\right)$ for every $F \subset L \subset \bar{F}$. Actually this is true for every representation unramified outside $S_{l}$ and to see this consider the localization exact sequence

$$
0 \longrightarrow H^{1}\left(G_{L, S_{l}} ; T_{l}\right) \longrightarrow H^{1}\left(G_{L} ; T_{l}\right) \stackrel{\oplus_{w \notin S_{l}} \partial_{w}}{\longrightarrow} \oplus_{w \notin S_{l}} H^{0}\left(g_{w} ; T_{l}(-1)\right)
$$

Note that $\partial_{w}$ can be factored as follows

$$
H^{1}\left(G_{L} ; T_{l}\right) \longrightarrow H^{1}\left(G_{w} ; T_{l}\right) \stackrel{\tilde{\partial}_{w}}{\longrightarrow} H^{0}\left(g_{w} ; T_{l}(-1)\right)
$$

On the other hand for $w \notin S_{l}$ by Lemma 2.8 (3) [BGK2] there is an exact sequence which comes from the restriction-inflation exact sequence

$$
0 \longrightarrow H_{f}^{1}\left(G_{w} ; T_{l}\right) \longrightarrow H^{1}\left(G_{w} ; T_{l}\right) \stackrel{\operatorname{Res}_{w}}{\longrightarrow} H^{1}\left(I_{w} ; T_{l}\right)^{g_{w}}
$$

In addition

$$
H^{1}\left(I_{w} ; T_{l}\right)^{g_{w}} \cong \operatorname{Hom}_{g_{w}}\left(I_{w} ; T_{l}\right) \cong \operatorname{Hom}_{g_{w}}\left(\mathbb{Z}_{l} ; T_{l}(-1)\right) \cong H^{0}\left(g_{w} ; T_{l}(-1)\right)
$$

and we check that the map $\operatorname{Res}_{w}$ can be identified with the boundary map $\tilde{\partial}_{w}: H^{1}\left(G_{w} ; T_{l}\right) \rightarrow H^{0}\left(g_{w} ; T_{l}(-1)\right)$. This shows that $H_{f, S_{l}}^{1}\left(G_{L} ; T_{l}\right) \cong H^{1}\left(G_{L, S_{l}} ; T_{l}\right)$. Now the Theorem follows by the next proposition.

Proposition 2.2. Let $\{1,2, \ldots, r\}=I \cup J$ be a partition, where $I=\left\{i_{1}, i_{2}, \ldots, i_{s}\right\}$ and $J=\left\{j_{1}, j_{2}, \ldots, j_{r-s}\right\}$. Given a fixed power $l^{M}$, there exist infinitely many places $v \notin S_{l}$ such that $r_{v}\left(\hat{P}_{i}\right)$ is an element of order at least $l^{M}$, for $i \in I$ and $r_{v}\left(\hat{P}_{j}\right)=0$, for $j \in J$.

Proof. We apply the method of the proof of Theorem 3.1 of [BGK3]. For the convenience of the reader we divide the proof into five steps. Define $\hat{\Lambda}_{I}$ and $\hat{\Lambda}_{J}$ in a similar way as $\hat{\Lambda}$. We define $\frac{1}{l^{k}} \hat{\Lambda}_{I} \quad\left(\right.$ resp. $\left.\frac{1}{l^{\infty}} \hat{\Lambda}_{I}\right)$ to be the submodule of $J_{f, S_{l}}\left(T_{l}\right)$, generated by all elements $\hat{R}$ such that $l^{k} \hat{R}=\hat{P}_{i}$ for some $i \in I$ with $k$ fixed (respectively, by all elements $\hat{R}$ such that $l^{k} \hat{R}=\hat{P}_{i}$ for some $i \in I$ and all $k$ ). The modules $\frac{1}{l^{k}} \hat{\Lambda}_{I}$ and $\frac{1}{l^{\infty}} \hat{\Lambda}_{I}$ are well defined by Prop. $1.1(1)$. Let $N_{k}$ (resp. $\left.N_{\infty}\right)$ be the subgroup of $G\left(\bar{F} / F_{l^{k}}\right)$ of such elements that act trivially on the submodule $\frac{1}{l^{k}} \hat{\Lambda}_{I} \quad\left(\right.$ resp. $\left.\quad \frac{1}{l^{\infty}} \hat{\Lambda}_{I}\right)$ of $J_{f, S_{l}}\left(T_{l}\right)$. Set $F_{l^{k}}\left(\frac{1}{l^{k}} \hat{\Lambda}_{I}\right)=\bar{F}^{N_{k}} \quad\left(\operatorname{resp} . \quad F_{l \infty}\left(\frac{1}{l^{\infty}} \hat{\Lambda}_{I}\right)=\bar{F}^{N_{\infty}}\right)$ and put 


$$
\begin{aligned}
& \mathcal{D}_{k}=G\left(F_{l^{k}}\left(\frac{1}{l^{k}} \hat{\Lambda}_{I}\right) / F_{l^{k}}\right) \\
& \mathcal{D}_{\infty}=G\left(F_{l^{\infty}}\left(\frac{1}{l^{\infty}} \hat{\Lambda}_{I}\right) / F_{l^{\infty}}\right) .
\end{aligned}
$$

Step 1. The argument in this step is as in [KP, Lemma 5]. By Proposition 1.3 there is an $m \in \mathbb{N}$ such that $l^{m} T_{l}^{s} \subset \Phi_{\hat{\Lambda}_{I}}^{(\infty)}\left(G\left(\bar{F} / F_{l \infty}\right)\right) \subset T_{l}^{s}$. Take $k \geqslant m$ and consider the following diagram:

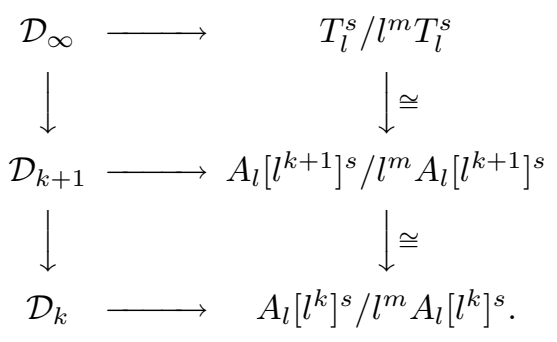

Observe that $\operatorname{ker} \Phi_{\hat{\Lambda}_{I}}^{(k)}=G\left(\bar{F} / F\left(\frac{1}{l^{k}} \hat{\Lambda}_{I}\right)\right)$ and $\operatorname{ker} \Phi_{\hat{\Lambda}_{I}}^{(\infty)}=G\left(\bar{F} / F\left(\frac{1}{l^{\infty}} \hat{\Lambda}_{I}\right)\right)$. Hence the horizontal arrows in diagram (2.3), induced by Kummer maps $\Phi_{\hat{\Lambda}_{I}}^{(\infty)}, \Phi_{\hat{\Lambda}_{I}}^{(k+1)}$, and $\Phi_{\hat{\Lambda}_{I}}^{(k)}$, are well defined. Now the definitions of these Kummer maps, given in section 1 , yield commutativity of the diagram (2.3) immediately. It is clear by an ascending chain argument that for $k \gg 0$ the image of the middle horizontal arrow is isomorphic to the image of the bottom horizontal arrow in diagram (2.3). Hence $\mathcal{D}_{k+1}$ maps onto $\mathcal{D}_{k}$ via the left bottom vertical arrow in (2.3) because for every $k$ the map $\mathcal{D}_{k} \rightarrow A_{l}\left[l^{k}\right]^{s}$, induced by Kummer map, is injective by definition of $\mathcal{D}_{k}$. So the tower of fields

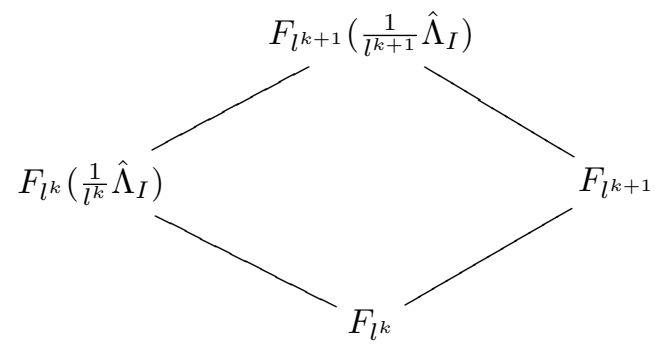

shows that for $k \gg 0$

$$
F_{l^{k}}\left(\frac{1}{l^{k}} \hat{\Lambda}_{I}\right) \cap F_{l^{k+1}}=F_{l^{k}}
$$

Step 2. By Proposition 1.3 we see that the upper horizontal arrow in the following commutative diagram has open image: 


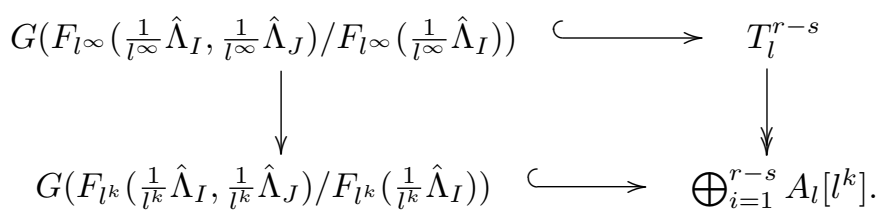

Hence the order of cokernel of the lower horizontal map in the diagram (2.6) is bounded independently of $k$. It follows that for $k \gg 0$ we can pick an element $\sigma \in G\left(F_{l^{k}}\left(\frac{1}{l^{k}} \hat{\Lambda}_{I}, \frac{1}{l^{k}} \hat{\Lambda}_{J}\right) / F_{l^{k}}\left(\frac{1}{l^{k}} \hat{\Lambda}_{I}\right)\right)$, with the property that all projections onto the $r-s$ summands $A_{l}\left[l^{k}\right]$ of the image of $\sigma$ via the bottom horizontal map in (2.6) have orders divisible by $l^{M}$.

Step 3. Consider the tower of fields:

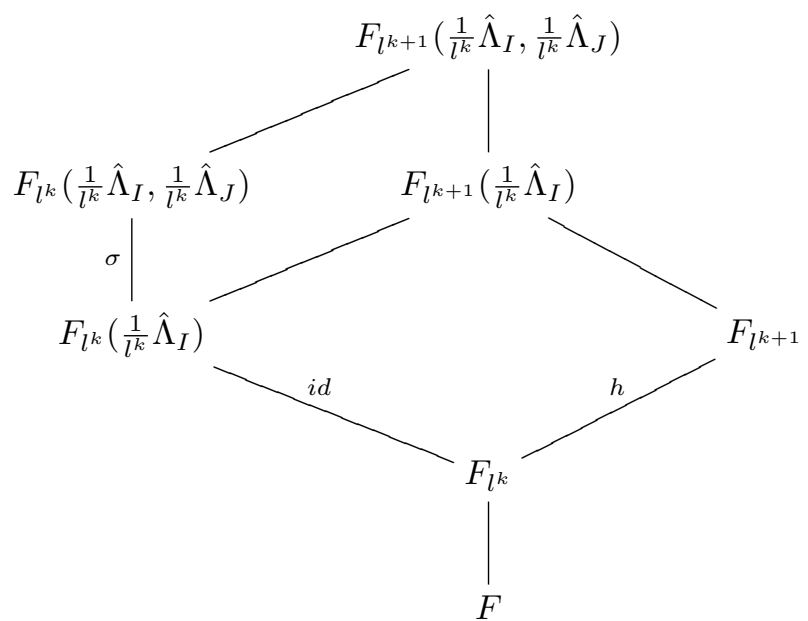

Take $k \gg 0$ such that $(2.5)$ holds and such that there is a nontrivial element $h \in G(\bar{F} / F)$ and such that $\rho_{l}(h)$ is acting on $T_{l}$ as a homothety $1+l^{k} u_{0}$, for some $u_{0} \in \mathbb{Z}_{l}^{\times}$. The element $h$ exists by [Bo, Cor. 1, p.702] in the number field case, and by [Za 3, Thm. 4.1] in the function field case. By abuse of notation we will denote the restriction of $h$ to $F_{l^{k+1}}$ also by $h$. Using the diagram (2.7) we construct an element

$$
\gamma \in G\left(F_{l^{k+1}}\left(\frac{1}{l^{k}} \hat{\Lambda}_{I}, \frac{1}{l^{k}} \hat{\Lambda}_{J}\right) / F_{l^{k}}\right) \subset G\left(F_{l^{k+1}}\left(\frac{1}{l^{k}} \hat{\Lambda}_{I}, \frac{1}{l^{k}} \hat{\Lambda}_{J}\right) / F\right),
$$

such that $\left.\gamma\right|_{F_{l^{k}}\left(\frac{1}{l^{k}} \hat{\Lambda}_{I}, \frac{1}{l^{k}} \hat{\Lambda}_{J}\right)}=\sigma,\left.\quad \gamma\right|_{F_{l^{k+1}}}=h,\left.\quad \gamma\right|_{F_{l^{k}}\left(\frac{1}{l^{k}} \hat{\Lambda}_{I}\right)}=i d$. By the Chebotarev density theorem [Ne, Theorem 6.4, p.132] there exist infinitely many prime ideals $v$ of $F$ such that $\gamma$ is equal to the Frobenius element for $v$ in the extension $F_{l^{k+1}}\left(\frac{1}{l^{k}} \hat{\Lambda}_{I}, \frac{1}{l^{k}} \hat{\Lambda}_{J}\right) / F$. For such a $v$ we fix a place $w$ in $F_{l^{k+1}}\left(\frac{1}{l^{k}} \hat{\Lambda}_{I}, \frac{1}{l^{k}} \hat{\Lambda}_{J}\right)$ above $v$.

Step 4. In Step 4 we prove that for $i \in I$ the point $\hat{P}_{i}$ maps to 0 via the bottom 
horizontal arrow in the diagram:

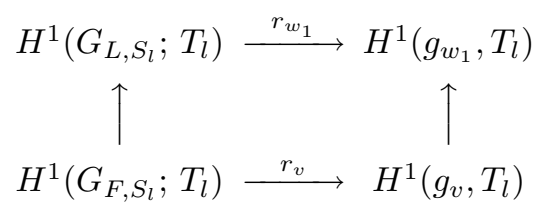

where $L=F_{l^{k+1}}\left(\frac{1}{l^{k}} \hat{\Lambda}_{I}\right)$ and $w_{1}$ is the place of $F_{l^{k+1}}\left(\frac{1}{l^{k}} \hat{\Lambda}_{I}\right)$ below $w$. The left vertical map in the diagram (2.8) is injective by Prop. 1.1 (3). The right vertical map is obviously injective. Let $l^{c_{i}}$ be the order of $r_{v}\left(\hat{P}_{i}\right)$ in $H^{1}\left(g_{v} ; T_{l}\right)$. The group $G\left(\bar{F} / F_{l^{k}}\left(\frac{1}{l^{k}} \hat{\Lambda}_{I}\right)\right)$ acts trivially on $\hat{Q}_{i}=\frac{1}{l^{k}} \hat{P}_{i} \in J_{f, S_{l}}\left(T_{l}\right)$ so by Prop. 1.1 (3) $\hat{Q}_{i} \in H^{1}\left(G_{F_{l^{k}}\left(\frac{1}{l^{k}} \hat{\Lambda}_{I}\right), S_{l}} ; T_{l}\right) \subset H^{1}\left(G_{L, S_{l}} ; T_{l}\right)$. Hence $\hat{Q}_{i}$ maps to the point $r_{w_{1}}\left(\hat{Q}_{i}\right) \in H^{1}\left(g_{w_{1}}, T_{l}\right)$ of order $l^{k+c_{i}}$. By the choice of $v$ we see that

$$
h\left(r_{w_{1}}\left(\hat{Q}_{i}\right)\right)=\left(1+l^{k} u_{0}\right) r_{w_{1}}\left(\hat{Q}_{i}\right),
$$

where $h$ is the element selected in Step 3. The choice of $v$ cf. diagram (2.7) implies that $r_{w_{1}}\left(\hat{Q}_{i}\right)$ comes from $H^{1}\left(g_{v}, T_{l}\right)$. Hence $h\left(r_{w_{1}}\left(Q_{i}\right)\right)=r_{w_{1}}\left(Q_{i}\right)$, so $l^{k} r_{w_{1}}\left(\hat{Q}_{i}\right)=0$ and $c_{i}=0$.

Step 5. We prove that for $j \in J$ the order of $r_{v}\left(\hat{P}_{j}\right)$ in $H^{1}\left(g_{v} ; T_{l}\right)$ is divisible by $l^{M}$ by computing its image in the bottom right corner of the following commutative diagram:

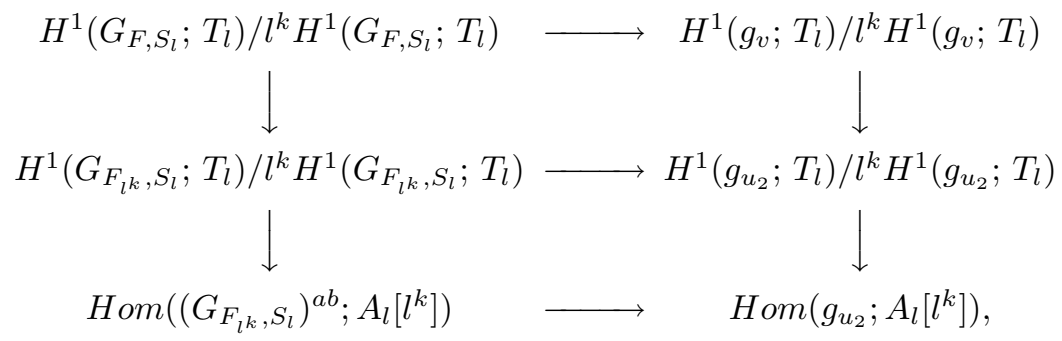

where $u_{2}$ is the place in $F_{l^{k}}$ below $w$, and $F_{l^{k}, S_{l}}$ denotes the maximal extension of $F_{l^{k}}$ contained in $\bar{F}$ and unramified outside of $S_{l}$. The image of $\hat{P}_{j}$ by the composition of left vertical maps is the homomorphism $\phi_{j}:\left(G_{F_{l k}, S_{l}}\right)^{a b} \longrightarrow A_{l}\left[l^{k}\right]$ induced by the Kummer map $\phi_{\hat{P}_{j}}^{(k)}$. Let $\tilde{\gamma}$ denote the extension to $F_{l^{k}, S_{l}}$ of the element $\gamma$ selected in Step 3. By the choice of $\sigma$ in Step 2 we obtain that $\phi_{j}(\tilde{\gamma})$ has order $\geqslant l^{M}$. The lower horizontal map in diagram (2.9) sends $\phi_{j}$ to a homomorphism whose value at the Frobenius element of $g_{u_{2}}$ has order at least $l^{M}$ by the construction of $\gamma$ and the choice of $v$ in step 3 . So the point $\hat{P}_{j}$ maps to a homomorphism of order at least $l^{M}$ in the lower right corner of the diagram (2.9) 


\section{References}

[BGK1] G. Banaszak, W. Gajda, And P. KRasoń On Galois cohomology of some p-adic representations and étale $K$-theory of curves, Contemporary Math. AMS 241, (1999) pp. 23-44

[BGK2] G. Banaszak, W. Gajda, And P. Krasoń Support problem for the intermediate Jacobians of l-adic representations, Journal of Number Theory 100, (2003), pp. 133-168

[BGK3] G. Banaszak, W. Gajda, And P. Krasoń Detecting linear dependence by reduction maps, MPI preprint 14, (2003), to appear in Journal of Number Theory

[Be] D. BERTRAND Galois representations and transcedence numbers, New advances in transcedence theory (Durham 1986), Cambridge Univ. Press (1988), pp. 37-55

[Bo] F.A. Bogomolov Sur l'algébricité des représentations l-adiques, C.R. Acad. Sci. Paris Sér. A-B, 290, (1980), pp. A701-A703

[De] P. Deligne La conjecture de Weil I, Publ. Math. IHES 43, (1974), pp. 273-307

[DF] W. Dwyer and E. Friedlander Étale K-theory and arithmetic, Trans. Amer. Math. Soc. 292 , (1985), pp. 247-280

[Fa] G. FALTings Endlichkeitssätze für abelsche Varietäten über Zalhkörpern, Inv. Math. 73, (1983) pp. 349-366

[FW] E. Friedlander And C. Weibel An Overview of Algebraic K-Theory, Proceedings of the Workshop and Symposium: Algebraic K-Theory and its Applications, H. Bass, A. Kuku, C. Pedrini editors, World Scientific, Singapore, New Jersey (1999) pp. 1-119

[J1] U. JANnsEn On the l-adic cohomology of varieties over number fields and its Galois cohomology, Galois groups over $\mathbb{Q}$. Proceedings of a Workshop Held March 23-27 1987, Y. Ihara, K. Ribet and J.P. Serre (eds), MSRI Publications 16, Springer Verlag, (1989), pp. 315-353

[KP] Ch. Khare, D. Prasad Reduction of homomorphisms mod $p$ and algebraicity, Journal of Number Theory 105, (2004), pp. 322-332

[Mi] J.S. Milne Étale cohomology, Princeton University Press, (1980)

[Ne] J. Neukirch Class Field Theory, Springer Verlag, (1986)

[Se] J.P. SERRE Sur les groupes de congruence des variéties abéliennes, Izvestija Akademii Nauk CCCP 35, (1971) pp. 731-735

[ST] J.P. SerRe, J. TATE Good reduction of abelian varieties, Annals of Math. 68, (1968) pp. 492-517 
[Za1] J.G. ZARHIN Isogenies of abelian varieties over fields of finite characteristics, Math. USSR Sb. 24, (1974), pp. 451-461

[Za2] J.G. ZARHIN A remark on endomorphisms of abelian varieties over function fields of finite characteristics, Math. USSR Izv. 8, (1974) pp. 477-480

[Za3] J.G. ZARHIN Torsion of abelian varieties in finite characteristic (in Russian), Matematiceskije Zametki 22, No. 1, (1977) pp. 3-11

This article is available at http://intlpress.com/HHA/v7/n3/a1/

G. Banaszak banaszak@amu.edu.pl

Mathematics Department

Adam Mickiewicz University

Poland

Poznań

ul.Umultowska 87

W. Gajda gajda@amu.edu.pl

Mathematics Department

Adam Mickiewicz University

Poland

Poznań

ul.Umultowska 87

P. Krasoń krason@sus.univ.szczecin.pl

Mathematics Department

Szczecin University

Poland

Szczecin

ul.Wielkopolska 15 